\title{
Intermédialités
}

Histoire et théorie des arts, des lettres et des techniques

Intermediality

History and Theory of the Arts, Literature and Technologies

\section{Le travail de la caméra : une pratique intermédiale. La conception de l'image du caméraman Eugen Schüfftan} (1886-1977)

\section{Karl Prümm}

Numéro 6, automne 2005

Remédier

Remediation

URI : https://id.erudit.org/iderudit/1005506ar

DOI : https://doi.org/10.7202/1005506ar

Aller au sommaire du numéro

Éditeur(s)

Centre de recherche sur l'intermédialité

ISSN

1705-8546 (imprimé)

1920-3136 (numérique)

Découvrir la revue

Citer cet article

Prümm, K. (2005). Le travail de la caméra : une pratique intermédiale. La conception de l'image du caméraman Eugen Schüfftan (1886-1977).

Intermédialités / Intermediality, (6), 65-78. https://doi.org/10.7202/1005506ar
Résumé de l'article

Cette étude vise à analyser le travail de la caméra, dans le cinéma narratif, en tant que pratique intermédiale, à partir de l'exemple d'un des plus grands opérateurs du $\mathrm{XX}^{\mathrm{e}}$ siècle, le caméraman Eugen Schüfftan. Après avoir offert un bref parcours de sa carrière artistique, l'analyse cherche à montrer la manière avec laquelle Schüfftan a développé une conception personnelle de l'image cinématographique, en s'appuyant sur les traditions de la peinture (Max Liebermann) et sur les expériences de la photographie contemporaine (László Moholy-Nagy, Otto Umbehr Umbo, Helmar Lerski). 


\title{
Le travail de la caméra: une pratique intermédiale.
}

\author{
La conception de l'image du caméraman \\ Eugen Schüfftan (1886-1977)
}

\author{
KarL PrüMm
}

L a mise en image de tout film qui se refuse à perpétuer simplement conventions et lieux communs donne nécessairement lieu à une pratique intermédiale. Elle demande une analyse et soulève un débat sur la conception de l'image héritée des autres médias. Jean-Luc Godard et son caméraman Raoul Coutard nous montrent dans leur film Passion (1982) ce qui d'ordinaire se passe plutôt discrètement et de façon camouflée: des comédiens rejouent les grands tableaux de l'histoire de l'art, nous les présentent comme des «tableaux vivants » qu'ils transfèrent dans la dimension du temps. Ils nous montrent que l'image du film ne peut se construire qu'en se référant aux autres médias, qu'elle est toujours un «entre-deux». Elle ne peut éclore sans cette dimension intermédiale.

Pour donner un autre exemple, nous pourrions dire que les images de l’Amérique que nous présente le caméraman Robby Müller dans les films de Jim Jarmusch - notamment Stranger than Paradise (1984), Down by Law (1986), Dead Man (1995) - proposent une exploration approfondie des photos du «Nouveau monde». Les images y sont là, anonymes, mais la référence au médium photographique n'en est que plus grande.

L'exploitation des grandes archives de l'image fait partie du quotidien lors de la production d'un film. Comme le rappelle a plusieurs reprises Henri Alekan dans son livre Des lumières et des ombres², les ordonnancements de la lumière dans les tableaux apparaissent souvent comme des sources d'inspiration et des «leçons» pour le cinéma. Le cadrage et la mise en lumière des grands photographes font

1. Ce texte a été traduit de l'allemand par Monique Prümm. La version finale a été établie par Philippe Despoix et André Habib.

2. Henri Alekan, Des lumières et des ombres, Paris, Librairie du Collectionneur, 1991 [1984], p. 189 . 
l'objet de discussions entre le metteur en scène et le caméraman avant que ne soient créées leurs propres images, leur atmosphère, leur ambiance, leur style.

Les artistes postmodernes, avec leur goût pour la citation et le simulacre, ont donné à cette pratique intermédiale un dynamisme nouveau. Le cinéma d'aujourd'hui est le lieu de transbordement des ordonnances de l'image et du caractère sensible des autres médias dont les caméramans deviennent les médiateurs et traducteurs. Pour se faire reconnaître, le caméraman doit de nos jours faire preuve d'une culture visuelle approfondie, tout autant que d'une large connaissance de l'histoire de l'art et de la photographie.

Le cinéma narratif exerce dès lors une remédiation multiple des arts visuels. La création et la concrétisation de ses figures amène dans presque tous les films à montrer des images (tableaux, gravures, photographies) qui en décorent habituellement les espaces privés. Des conventions culturelles sont ainsi mises en images, certaines traditions de la peinture et de la photographie gagnent une nouvelle présence, fût-ce de façon indirecte. Cette remédiation des grands maîtres et des œuvres célèbres, qui tombe plutôt sous la responsabilité du directeur artistique, peut donner lieu à un jeu de références ou à une utilisation précise des signes visuels, comme c'est le cas par exemple dans les films de Hitchcock.

Mais le regard de la caméra permet aussi une nouvelle perception filmique des objets d'art. Dans la séquence finale de Andrej Rubljow (1966) d'Andrei Tarkovski, la caméra tâte la surface d'un tableau que l'on attribue à Andrej Rubljow, peintre d'icônes du Moyen Âge. Le film glisse à ce moment de la fiction vers l'objectivation du tableau. La caméra dépasse les limites du cadre et entreprend un voyage presque interminable à l'intérieur du tableau, créant par ses mouvements une nouvelle exploration du regard, mettant en œuvre son propre assemblage de significations.

Durant la séquence finale, Tarkovski limite sciemment sa remédiation filmique à la peinture d'icônes. Il sépare de façon stricte cette apothéose de l'art chrétien de la narration biographique, renonçant ainsi à effectuer un rapprochement avec les motifs et le scénario de la vie de Rubljow.

Le film The Girl with a Pearl Earring (Peter Webber, 2003), où la création d'un des portraits les plus mystérieux de Jan Vermeer est raconté et interprété, procède tout autrement et suit le chemin plus traditionnel du récit des films d'artistes. Le modèle sort ici du tableau et entre dans l'espace d'une narration qui imagine la genèse de ce portrait, où tous les détails et les gestes de l'image trouvent leur explication concluante, leur lieu concret. Le film ne se réfère pas seulement à ce tableau. Ses lieux et ses intérieurs sont tirés des tableaux très détaillés du maître hollandais, jusque dans les moindres accessoires. La peinture 
de Vermeer se voit ici soumise à une double remédiation. D’une part, la création de l'image se transforme au fil du récit. D'autre part, le portrait de la jeune fille s'adapte aux conventions de la présentation et aux modi de la représentation du cinéma. Il est transformé dans de nouveaux espaces de l'image et dans de nouveaux ordres du regard.

Dans ce processus très ambitieux et à plusieurs dimensions, le caméraman - il s'agit d'Eduardo Serra - joue un rôle central. C'est lui qui est responsable de la mise en images, c'est lui qui remet en scène les tableaux de Vermeer en se servant des moyens du cinéma. Il traduit les pigments et l'application des couleurs en opérations photographiques. C'est à lui que revient la tâche de poser la lumière pour que l'émulsion du matériel filmique rende les effets du tableau d'origine et son atmosphère.

Malgré tout cela, il est curieux de constater que le travail de la caméra n’ait pas encore été l'objet des recherches et des réflexions sur l'intermédialité. Les raisons de ce déficit sont multiples. Tout d'abord, le travail du caméraman est souvent sous-estimé. Il s'agit d'un travail fonctionnel et le caméraman est membre d'un ordre hiérarchique. Bien que ce soit lui qui mette sa fantaisie de l'image, son intérêt pour l'image, son orientation de l'image au service du texte, il ne fait bien souvent que des propositions au metteur en scène, qui les acceptera ou les modifiera à son gré. La subjectivité du caméraman devient invisible dans le texte filmique. Son influence est certes déterminante pour le texte, puisqu'il lui donne sa forme photographique, puisqu'il réalise et surveille son façonnement il demeure qu'il ne domine pas ce texte. De plus, le public et la critique, prisonniers de la théorie de l'auteur, ne prennent en considération que le metteur en scène comme auteur-créateur du texte filmique, comme inventeur et conteur.

Mais il ne faudrait pas penser sauver la subjectivité du caméraman en substituant à la politique de l'auteur celle du caméraman. Il s'agit plutôt de porter un regard novateur sur le processus d'invention de l'image et de sa réalisation. Il s'agit d'expliquer la mise en image comme un façonnement, un acte photographique. Car il peut arriver qu'un caméraman agisse dans le processus de création ordinaire de l'image avec une telle personnalité, une telle assurance, que son travail va conserver une griffe individuelle, aussi diverses que soient les constellations de son travail. Eugen Schüfftan est un tel caméraman³: il apporte avec lui

3. Sur Eugen Schüfftan, voir Karl Prümm, «Peindre et raconter avec la lumière: de l'esthétique de la caméra de Mademoiselle Docteur (1937)», Archives, Perpignan, Institut Jean Vigo, n 73 , décembre 1997, p. 12-16; Helmut G. Asper (éd.), Nachrichten aus Hollywood, New York und anderswo: der Briefwechsel Eugen und Marlise Schüfftans mit Siegfried und Lili Kracauer, Trier, Wissenschaftlicher Verlag, 2003. 
un véritable programme de l'image. Il offre sa propre vision et ne fait pas que se plier aux exigences du récit et du metteur en scène.

Né en 1886 (et non en 1893 comme le notent les encyclopédies), Eugen Schüfftan est marqué par la culture du film sous la République de Weimar. Bien plus, il est défini par elle. À cette époque, on assiste à une véritable prise de conscience du travail de la caméra. Les caméramans allemands étaient alors estimés de par le monde en raison de leurs raffinements techniques et de leur inventivité (il suffit de penser à Karl Freund ou à Carl Hoffmann). Ils s'étaient organisés et avaient fondé le Klub der Kameraleute Deutschlands pour pouvoir représenter et défendre leurs intérêts de façon plus efficace. Certains, comme Franz Planer, demandent publiquement la parole et exigent une meilleure prise en considération des nécessités de la caméra lors de la production du film: «Écoutez le caméraman 4 !»

Dans les années 1920, on voit naître en Allemagne un intérêt du public pour la spécificité de la caméra. C'est ainsi que des revues comme Kameratechnik ou Filmtechnik discutent des problèmes et des nouveautés techniques - du matériel d'enregistrement jusqu'aux possibilités du mouvement de la caméra. Mais il se développe aussi une critique du film sous l'angle technique de la caméra. On commence à le juger sous celui de la visualité, de la forme photographique. Ces débats intensifs sur le rôle de la caméra ont beaucoup contribué à accorder à la photographie de film une dimension professionnelle. Ils ont ouvert la «sphère de l'intermédiatique ».

Jusqu'au milieu des années 1920, on considérait le tableau peint comme la catégorie de référence par excellence du «visuel » et les caméramans suivaient une esthétique traditionnelle de l'image. Ils en assombrissaient les coins pour retrouver l'obscurité de la peinture de salon du XIX ${ }^{\mathrm{e}}$ siècle. Les articles de presse spécialisée enseignaient comment arriver à l'aide de voiles et de filtres à des effets poétiques, enlevant à la photographie sa netteté prosaïque. Or, dans la seconde moitié des années 1920, la photographie devient de plus en plus présente dans la vie quotidienne, dans les journaux, dans la presse illustrée, dans la publicité. Elle va peu à peu relayer la peinture. En 1929, l'exposition légendaire du Werkbund à Stuttgart, Film und Photographie, montre bien le changement de paradigme. Les deux mouvements avant-gardistes sont montrés côte à côte et révèlent les interdépendances entre personnes et techniques. Le tableau peint n'est plus la

4. Franz Planer, «Hört auf den Kameramann », Film-Kurier, 19 octobre 1929, repris dans Rolf Aurich, Wolfgang Jacobsen (dir.), Werkstatt Film. Selbstverständnis und Visionen von Filmleuten der zwanziger Jahre, München, Édition Text + Kritik, 1998, p. 97-98. 
référence première pour la cinématographie. La photographie prend sa place, avec ses contrastes durs et clairs.

Dans ce monde en mouvement des années 1920, Schüfftan joue un rôle exceptionnel. Il est le médiateur entre les divers médias, il unit et synthétise les différents arts. Il commence sa carrière comme peintre, devient architecte après la guerre, puis décorateur. En 1920, il arrive au film. Son intérêt pour le média est au départ d'ordre technique. En 1923, il invente en bricoleur le système Schüfftan, un procédé qui consiste à l'aide d'un miroir à combiner des objets réels à des modèles miniatures5. Les années suivantes seront consacrées à perfectionner son invention et ce n'est qu'en 1930 qu'il réalise son rêve. À l'âge de 44 ans, il commence son travail de caméraman sur le film Menschen am Sonntag (Les hommes du dimanche, 1930, Curt Siodmak, Robert Siodmak, Edgar G. Ulmer, Fred Zinnemann), un film entièrement réalisé en dehors de l'industrie. Les nazis arrivent au pouvoir alors que la réputation de Schüfftan comme caméraman commence à s'établir. Il se voit obligé de quitter l'Allemagne à cause de son origine juive. Ceci provoque une rupture dans sa carrière et les événements politiques vont dès lors compromettre la continuité dans son travail. Schüfftan ne réussit à s'établir nulle part. C'est néanmoins en France, au courant des années 1930, que son esthétique de l'image attire l'attention et commence à être estimée. Les coopérations avec les metteurs en scène allemands exilés Georg Wilhelm Pabst, Robert Siodmak et Max Ophüls, mais surtout celles avec Marcel Carné et Jacques Prévert, vont être au centre de son œuvre. En 1940, Schüfftan essaie de s'établir aux États-Unis où il connaît une période sombre dans son travail. La toute-puissante association ASC (American Society of Cinematographers) refuse de l'admettre. Il lui faut donc travailler «undercover» comme «supervisor» et non comme chef opérateur. Mise à part la coopération avec Georges Franju - La tête contre le mur (1959) et Les yeux sans visage (1960), qui lui attirent une renommée internationale - dans les années 1950, Schüfftan tourne, surtout en France, des films de moindre importance. En 1961, on lui décerne l'Oscar dans la catégorie «Photographie noir et blanc » pour le film The Hustler (Robert Rossen), une reconnaissance tardive de son mérite.

Les nombreuses césures et transitions auxquelles Schüfftan a dû faire face au cours de sa carrière n’ont pas été seulement une expérience douloureuse, elles ont conduit à la réflexivité propre à son travail. Il dut maintes fois repartir à zéro, se redéfinir. Mais le peu de déclarations que l'on a de lui montrent une étonnante continuité de principe. Le vocable avec lequel il opère ne manque pas

5. Ce système a en particulier été employé dans le film Metropolis (1927) de Fritz Lang. 
d'emphase: le caméraman est pour lui «l'homme de l'image». Le travail de la caméra fait partie d'une histoire universelle de l'image, si bien que le caméraman doit disposer d'une connaissance souveraine des expériences et des traditions de l'image des autres arts. Pour lui, le travail de la caméra, c'est le travail de l'image, comme élargissement de ses possibilités d'expression. Selon Schüfftan, le cinéma accomplit à la perfection un achèvement de l'image, il dépasse les limites de la bidimensionnalité :

Tout comme la musique, l'image de film connait sa grande expression avec la troisième dimension et par elle, dans le symphonique. Les peintres de tous temps ont désiré s'exprimer avec cette troisième dimension, de Rembrandt aux peintres abstraits d'aujourd'hui. L'imagination vient de la troisième dimension et vit en elle ${ }^{6}$.

On retrouve ici une théorisation du travail de la caméra d'une grande portée. Selon Schüfftan, c'est dans l'image filmique, où se retrouvent tous les arts, que les désirs d'expression des peintres vont pouvoir se réaliser. Une troisième dimension, jusque-là réservée à la musique, s'ouvre grâce au cinéma: la dimension du mouvement et du temps. L'image filmique est implicitement définie comme image-mouvement et image-temps. Mais surtout, elle est l'expression de l'imagination, elle est le résultat d'une imagination subjective. Elle est par là bien loin du principe de la reproduction mécanique et de la photographie réaliste.

Le passage des frontières, le passage d'une cinématographie à l'autre, amènent un caméraman aussi ambitieux que Schüfftan à résumer ses possibilités et à affirmer sa singularité. C'est ce qu'il réussit, manifestement sans peine, durant son exil en France. Les critiques parlèrent rapidement d'une «lumière allemande» qui donnait à son œuvre un rayonnement particulier7. C'est sur cette nouvelle identité retrouvée qu'il attira, d'après une lettre de juin 1941, l'attention de son agent Paul Kohner qui négociait à Hollywood, soulignant la différence entre son travail et les conventions du cinéma narratif:

Je représente une forme particulière du travail de la caméra et je ne pense pas que vous ayez suivi mes travaux en France ces dernières années. Depuis environ cinq ans, j'ai pris un chemin que, par exemple, [Gregg] Toland prend aujourd'hui (je ne connais pas particulièrement les autres opérateurs d'Hollywood). C'est par l'image

6. Eugen Schüfftan interviewé par Werner Zurbuch. Voir Werner Zurbuch, «Eugen Schüfftan-Meister der Filmtechnik», Filmtechnikum, München, nº 6, juin 1962, p. 179180 (trad. Monique Prümm).

7. Voir Philippe Roger, «L’obscure clarté: la lumière allemande du cinéma français», dans Heike Hurst, Heiner Gassen (dir.), Tendres ennemis: cent ans de cinéma entre la France et l'Allemagne, Paris, Editions L'Harmattan, 1991, p. 111-137. 
que j’essaie de donner caractère au scénario, de soutenir le travail du film et de faire ressortir les différentes scènes. J'ai bien réussi en France avec cette sorte de photographie et je crois que c'est la seule façon de travailler pour un caméraman. Je sais que cela contredit la manière hollywoodienne, puisqu'on n'aime pas que le caméraman s'exprime par son travail. Ce n'est que maintenant qu'on se rappelle des possibilités de l'image grâce au film Citizen Kane ${ }^{8}$.

C'est la lumière qui va donner aux images de Schüfftan leur langage, leur expression spécifique. Ce sont des «images-lumière », dans un sens très élémentaire. Elles renouvellent par là l'origine du média photographique, qui est enregistrement et conservation de la lumière, pour renouer en même temps avec l'avant-garde de Weimar. Ce retour au principe original du photographique était surtout appelé par László Moholy-Nagy: «Le photographe est le sculpteur de l'image, la photographie est le modelage de la lumière9 ${ }^{\text {. » }}$

Moholy-Nagy voulait libérer la photographie de ses fonctions et de ses techniques traditionnelles, de sa prétention au document, du réalisme de surface, mais aussi de la perspective centrale et du regard humain. Sa conception de la photographie sans caméra le mène en toute conséquence au photogramme, à une image sans objet, à une pure modulation de la lumière. Sa remarque de 1925 à propos «des éléments essentiels de la réalisation du film», longtemps négligés au profit de la «reproduction d'actions dramatiques», pourrait se lire comme la caractérisation du travail de Schüfftan pendant les années 1930: «Les tensions de la forme, de l'infiltration, des rapports clair-obscur, du mouvement, du tempo ${ }^{10}$.»

Pour Schüfftan, chaque prise de vue est un monde de lumière soigneusement élaboré. La lumière n'est pas seulement un moyen pour illuminer un monde, c'est l'élément central de la création de l'image. Dans ses films des années 1930, on ne trouve qu'un éclairage artificiel, posé et dominé par le caméraman. La lumière naturelle en est exclue. Les images de Schüfftan ne sont pas des reproductions de l'existant, ce sont des créations pleines de la lumière imaginée par le caméraman.

8. «Lettre de Eugen Schüfftan à Paul Kohner », 31 juin 1941, conservée aux Archives de la Fondation cinématographique allemande (Filmmuseum), Berlin (trad. Jacqueline Parodi).

9. László Moholy-Nagy, «Fotografie ist Lichtgestaltung» [1928], cité dans Andreas Haus, Moholy-Nagy, Fotos und Fotogramme, München, Schirmer-Mosel Verlag, 1978, p. 75 (trad. Monique Prümm).

10. Moholy-Nagy, Malerei Photographie Film, Bauhausbuch Nr 8, München, Albert Langen, 1925, p. 32 (trad. Monique Prümm). 


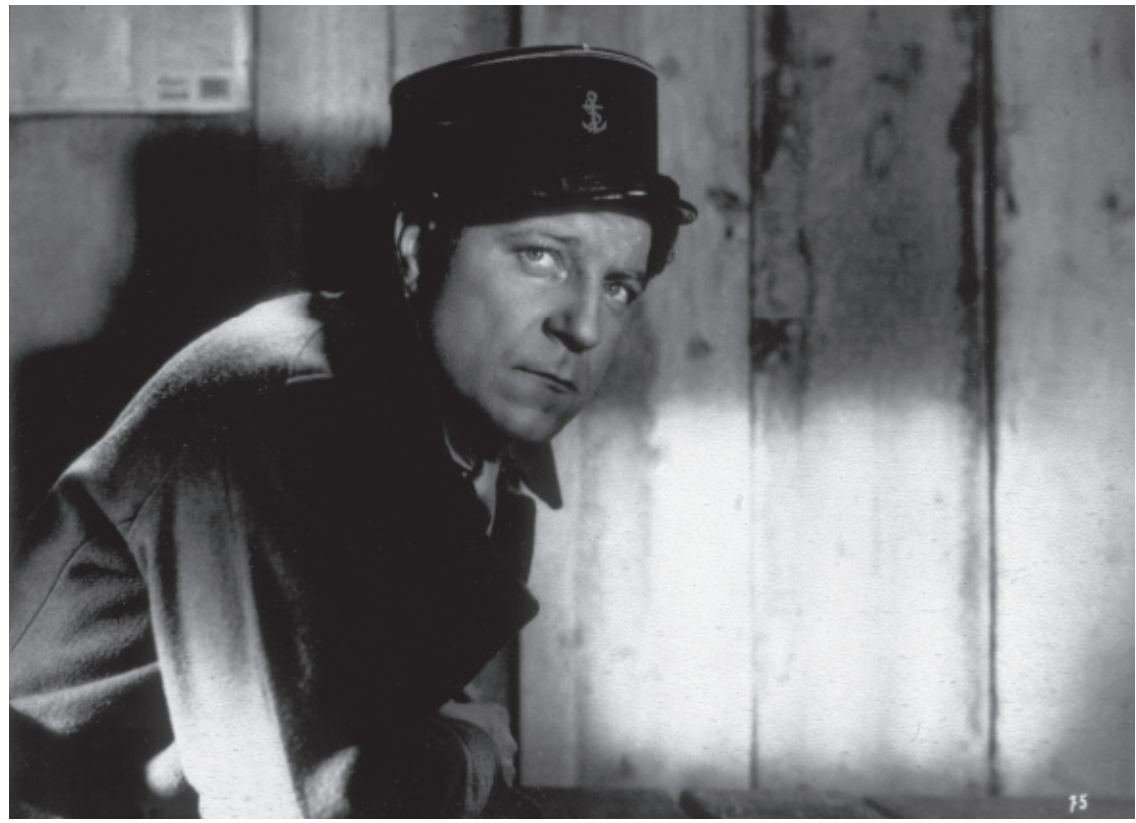

Fig. 1. Jean Gabin dans Le quai des brumes (Marcel Carné, 1938). Source : Henri Alekan, Des lumières et des ombres, Paris, Librairie du Collectionneur, 1991 [1984].

Dans ces «images-pensées », la distinction entre intérieur et extérieur est abolie. Les deux sphères sont représentées par la lumière artificielle. Dans Le quai des brumes (Marcel Carné, 1938) par exemple, l'extérieur n’est pas un paysage avec dégradés et gradations, mais une confrontation de clairs et d'obscurs, un chiaroscuro radical.

Pour les intérieurs, Schüfftan utilise une lumière éblouissante qui produit une surexposition, voire une déformation. La lumière latérale crue brise le cadrage, déforme le corps de Jean Gabin et trempe le dessus de son uniforme dans un blanc criard (fig. 1). La lumière frontale a un effet encore plus fort, elle est brûlure, agression sur l'image. L'arrière-plan semble se dissoudre et il est en même temps utilisé comme réflecteur. La lumière réfléchie éclaire indirectement le visage de Jean Gabin et permet de déchiffrer l'expression de sa physionomie. Schüfftan intègre avec beaucoup d'art ses jeux de lumière à la narration. Il «soutient», comme il dit, il «force » la percée du scénario. Et pourtant ce monde de lumière a une valeur autonome, crée une matérialité originale.

Les ordonnancements de lumière dans ces images ne sont pas compréhensibles à première vue. Les sources de lumière ne sont pas visibles, on ne peut pas 


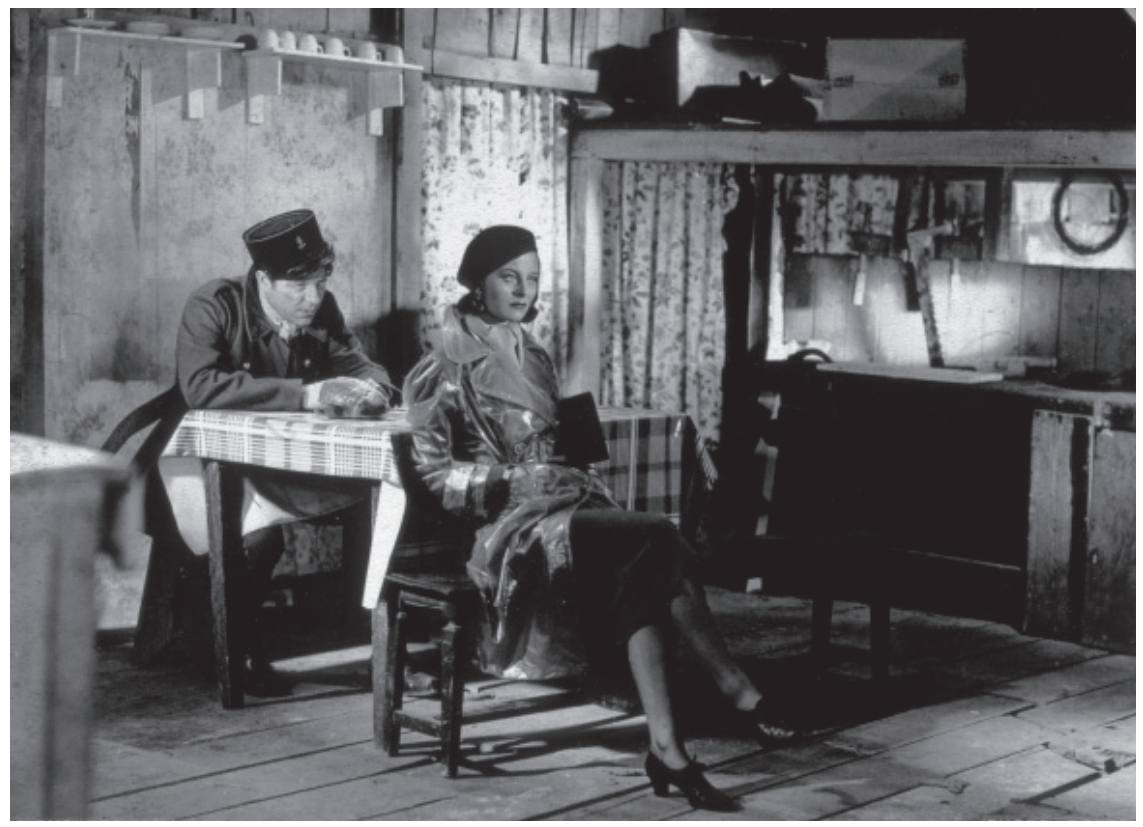

Fig. 2. Jean Gabin et Michèle Morgan dans Le quai des brumes. Source: Henri Alekan, Des lumières et des ombres, Paris, Librairie du Collectionneur, 1991 [1984].

les localiser. Elles font de l'espace un labyrinthe où il est difficile de distinguer le centre de la périphérie, l'essentiel du secondaire. Tout l'espace de l'image devient un plan expressif et le spectateur se transforme en constructeur dans un horizon de significations ouvert et fluide. La lumière qui échappe à tout code clair, et les ombres, totalement inextricables, créent un mouvement qui correspond exactement au discours des sexes (fig. 2). «Hommes et femmes ne se comprendront jamais », s'exclame Jean Gabin, "parce qu'ils vivent dans des mondes différents. » Mais ses paroles agressives naissent d'un mouvement intérieur qu'il ne s'avoue pas encore: la convoitise.

Schüfftan a créé de cette manière une nouvelle forme de l'image, un « décentrage », une «dé-subjectivation » de l'image. Le corps et le visage humains ne sont plus le centre de la mise en scène. La lumière attire le regard sur d'autres foyers. Il arrive très souvent que le visage des acteurs soit plongé dans une semiobscurité ou que ceux-ci apparaissent en silhouette. Le chiaroscuro plat à structures géométriques fait déjà penser aux images du film noir. Schüfftan est bien un pionnier. Il a développé la lumière de direction pour en faire un système d'éclairage, praticable et réglable. 


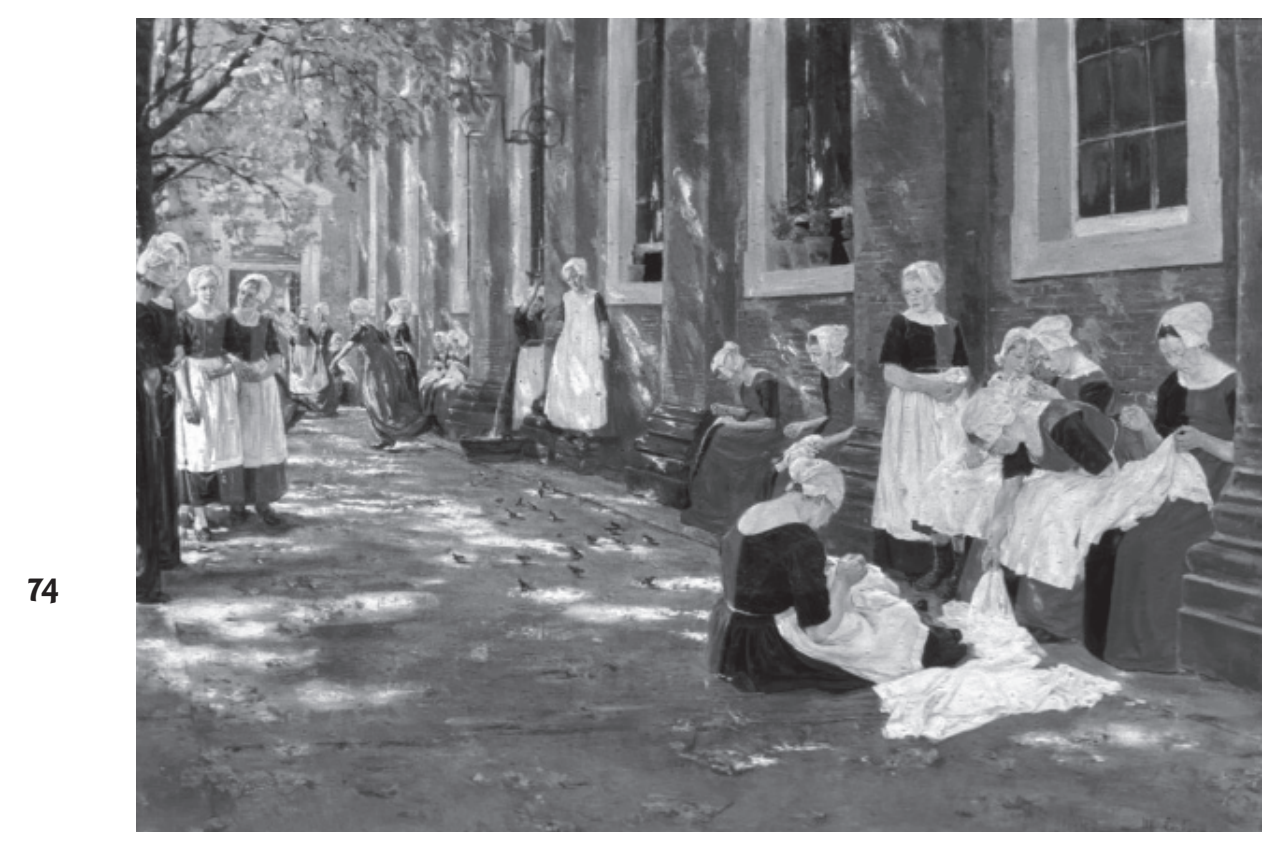

Fig. 3. Max Liebermann, Freistunde im Amsterdamer Waisenhaus (1882), Städelsches Kunstinstitut Frankfurt am Main. Source: Max Liebermann, Jahrhundertwende, Angelika Wesenberg (dir.), Berlin, Nicolaische Verlagsbuchhandlung, 1997.

Il faut aussi noter qu'il a de toute évidence actualisé les grands tableaux de la peinture. Cette lumière latérale arbitraire qui se glisse si durement avait déjà été la marque de Caravaggio. Il l'avait maîtrisée à un tel degré de perfection qu'on pourrait presque parler de spotlight. Schüfftan, le peintre, connaissait sans aucun doute ces mises en scène de la lumière.

Les impressionnistes français ont aussi joué un grand rôle dans sa prise de conscience de la lumière et de ses différents aspects. L'idée fondamentale que se fait Schüfftan de l'image filmique comme produit subjectif, comme produit de l'imagination, renvoie à cette tradition. Il est possible que ce soit Max Liebermann qui lui ait fait connaître les impressionnistes français. Dans ses études du monde social, aux environs de 1900, il a donné à la lumière son caractère d'objet, ouvrant ainsi les possibilités pour la narration figurative dans le film.

Schüfftan connaissait certainement les tableaux de Max Liebermann. Aux environs de 1910, ce premier était en relation avec la Berliner Secession. Si l'on en croit ses déclarations (non vérifiables), il y aurait exposé ses premiers tableaux. Liebermann, qui appartenait pendant ces années-là au milieu artistique de 


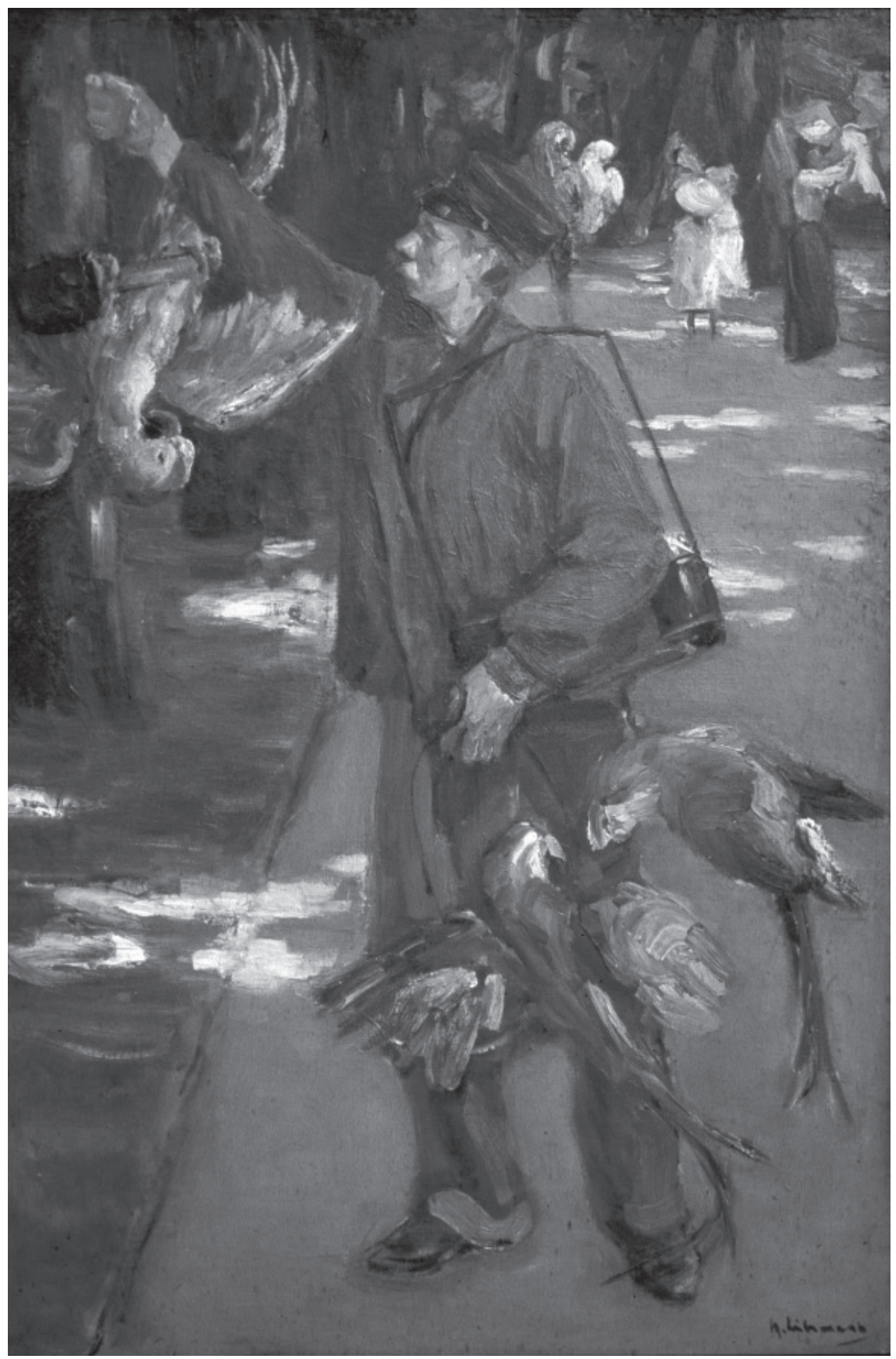

Fig. 4. Max Liebermann, Der Papageienmann (1902), Museum Folkwang Essen. Source: Max Liebermann, Jahrhundertwende, Angelika Wesenberg (dir.), Nicolaische Verlagsbuchhandlung, Berlin, 1997. 
Berlin, était en effet l'un des initiateurs des expositions de la Secession. Un jeune peintre comme Schüfftan, qui avait l'ambition de réussir dans ce milieu, ne pouvait donc manquer de s'intéresser à un modèle aussi puissant.

Bien des tableaux de Liebermann sont des images de lumière, comme par exemple Freistunde im Amsterdamer Waisenhaus (1882, fig. 3). L'image s'ouvre sur l'arrière-plan, vers la lumière. Le premier plan est dominé par l'ombre et le mur sombre. Mais des ronds clairs lumineux y montrent aussi l'ardeur du soleil et donnent ainsi mouvement et vie à l'ensemble. Le blanc des robes, des fichus et des coiffes reflète la lumière de façon diverse et graduée. En virtuose, Liebermann use du blanc des accessoires comme d'un lieu de rassemblement et de réflexion de la lumière qui tombe à travers les arbres, pour créer ainsi des surfaces élargies de lumière.

Ces taches accentuées prennent un caractère de spot qui intervient avec sa lumière dure et criarde dans l'image, comme par exemple dans Der Papageienmann (1902, fig. 4). Ceci annonce la lumière brûlante de Schüfftan. Vers 1900, Liebermann préférait les espaces de lumière clairement structurés: des taches lumineuses animent un premier plan ombragé, l'arrière-plan est baigné de lumière.

Laccentuation expressive de la lumière du soleil souligne la dimension du temps, le caractère du moment et de l'impression unique de l'image. En même temps, le fait d'étaler les couleurs si distinctement rend visible le procédé. La technique devient réflexion consciente. Les couleurs se détachent des objets et acquièrent leur propre vie qui laisse pressentir un ordre abstrait. Le caractère instantané de l'image et la prise de conscience de la technique de la peinture sont les deux éléments de l'art de Liebermann, vers 1900, que l'historien de l'art Thomas W. Gaethgens interprète comme une influence directe des impressionnistes français, en particulier de Manet et de Degas ${ }^{11}$.

Dès ses débuts comme caméraman, dans Menschen am Sonntag, Schüfftan a souvent construit de telles images. Un premier plan ombragé suggère au spectateur des images de la ville en été sous le soleil. La référence aux techniques des impressionnistes est sensible. Mais dans ce film, le façonnement par la photographie de l'époque est encore plus visible. Celui-ci est une expérience photographique dans toute sa totalité. Dans une célèbre séquence, au milieu du film, on voit un photographe travailler sur la plage de Wannsee. La caméra du

11. Thomas W. Gaethgens, «Liebermann und der Impressionismus », dans Angelika Wesenberg (dir.), Max Liebermann Jahrhundertwende, Berlin, Nicolaische Verlagsbuchhandlung, 1997, p. 141-152. 


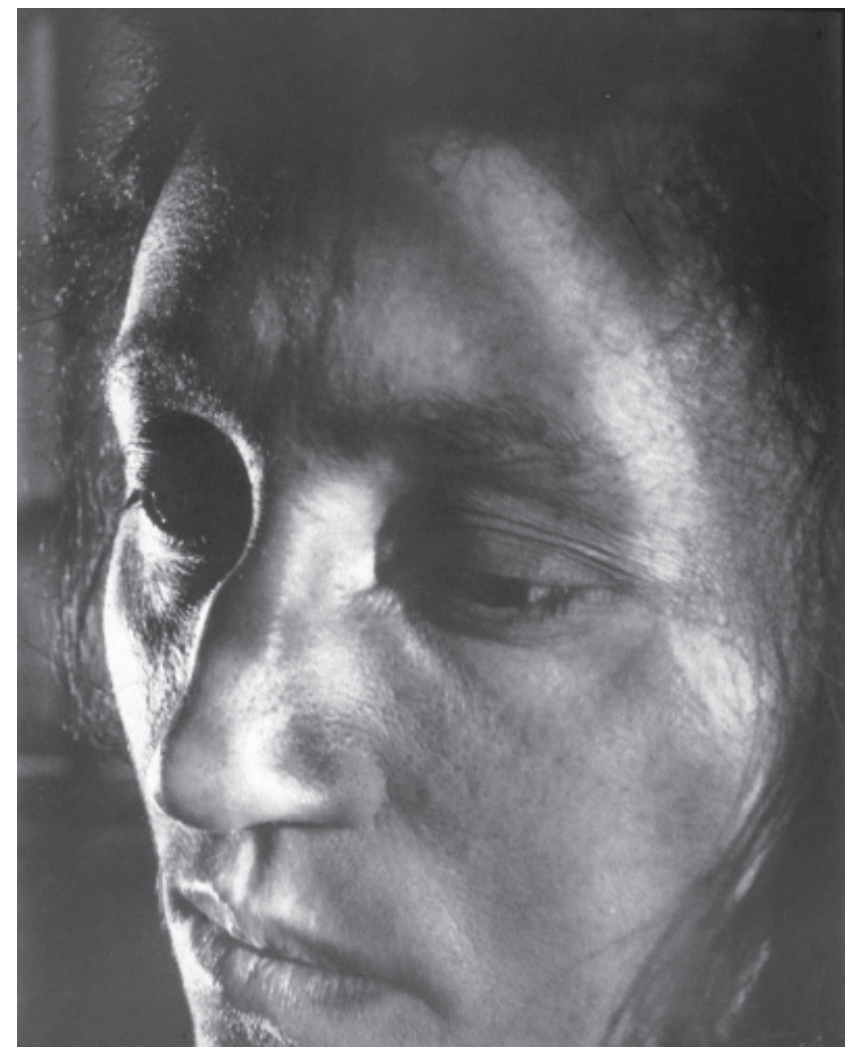

Fig. 5. Helmar Lerski, Reinemachefrau. Köpfe des Alltags, Berlin, Hermann Rockendorf Verlag, 1930. Source: Ute Eskilden, Jan-Christopher Horak, Helmar Lerski. Lichtbilder, Fotografien und Filme 1910-1947, Essen, Das Museum, 1982. Catalogue d'exposition du Folkwang-Museum.

film et l'appareil du photographe deviennent identiques. La photo du film se fige pour devenir photo, puis s'anime à nouveau pour redevenir photo-mouvement. C'est ce qu'on peut appeler l'autoréférence de Menschen am Sonntag.

Ce film tient du geste de photographe-portraitiste. Il nous présente une galerie de portraits de jeunes citadins. Peu avant sa sortie, Schüfftan avouait avoir fait ce film avec le plus grand plaisir, car il avait eu l'occasion de "photographier des dilettantes, des enthousiastes du cinéma comme il les voyait ${ }^{12}$.» Au-delà des

12. Eugen Schüfftan, «Der Mann des Spiegel-Trickverfahrens», Film-Kurier,

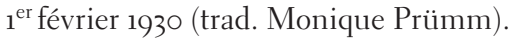


images conventionnelles, le photographe peut donc mettre son propre regard en valeur, ses intérêts de l'image et ses préférences. Schüfftan reprend ainsi les expériences du Neue Portrait des années 1920 si clairement qu'on est tenté de parler de citation. Par les opérations de lumière, la physionomie du modèle devient le matériel du photographe. Schüfftan transfère ses nouvelles techniques dans le film. Pour lui aussi, le portrait n'est plus le miroir de l'âme, mais le résultat d'une construction photographique, d'un jeu avec la lumière. Il reprend les impulsions d'Otto Umbehr Umbo qui avait introduit le chiaroscuro dans la photographie de portrait et avait, presque avec violence, transformé les physionomies en surfaces noir et blanc.

Il reprend plus particulièrement Helmar Lerski, qu'il appelait son «Kampffreund», son ami de combat dans la recherche d'une expression photographique neuve. Lerski s'approche extrêmement des visages de ces Têtes de tous les jours (Köpfe des Alltags, tel est le titre de son livre paru en 1930 avec 80 portraits) ${ }^{13}$. Il concentre dans des miroirs les faisceaux lumineux du soleil et les renvoie sur les visages, ce qui donne un effet singulier de raideur et de majesté héroïque (fig. 5). Schüfftan réutilise cette technique dans Menschen am Sonntag, créant ainsi une texture qui sort presque de la narration et de la mise en scène.

Les stratégies intermédiales de Schüfftan sont un exemple du jeu qu'ouvre le travail de la caméra entre les médias. Schüfftan était un pionnier à tous points de vue. C'est l'un des premiers caméramans dont le travail de l'image fasse preuve d'une «conscience intermédiale » et qui s'approprie d'une nouvelle manière les traditions de la peinture et de la photographie. Il a fait de la lumière et des techniques d'éclairage un instrument capable de modulations. C'est cette double compétence qui a amené Henri Alekan à voir en Schüfftan « un grand maître » auprès duquel il fit son apprentissage et dont il s'est senti l'élève toute sa vie ${ }^{14}$.

13. Helmar Lerski, Köpfe Des Alltags, Berlin, Hermann Rockendorf Verlag, 1931.

14. «À la mémoire d'Eugen Schüfftan qui m’a révélé l'art de l'image et de Lotte Eisner qui m’a incité à écrire ce livre. » Telle est la dédicace du livre Des lumières et des ombres d'Henri Alekan. 